\section{AB0548 ASSESMENT OF THE PHYSICAL ACTIVITY IN SYSTEMIC SCLEROSIS PATIENTS BY USING COMMERCIAL SMART BANDS AND ITS ASSOCIATION WITH DISEASE CHARACTERISTICS: A PILOT STUDY}

A. Avanoğlu Güler ${ }^{1}$, H. Karadeniz ${ }^{1}$, R. Bilici Salman ${ }^{1}$, H. Satıs ${ }^{1}$, H. Babaoglu ${ }^{1}$, N. Atas ${ }^{1}$, S. Haznedaroglu', B. Goker ${ }^{1}$, M. A. Ozturk ${ }^{1}$, A. Tufan ${ }^{1} .{ }^{1}$ Gazi University Faculty of Medicine, Department of Rheumatology, Ankara, Turkey

Background: Systemic sclerosis (SSc) is a complex disease, characterized by multi-system organ involvement including interstitial lung disease (ILD) and pulmonary arterial hypertension (PAH). The decrease in physical activity in SSc patients with lung involvement has been demonstrated by self-reported physical capacity and 6 min-walking test $(6 \mathrm{MWT})(1,2)$. Commercial smart bands can provide data on daily physical activity, sleep characteristics, blood oxygen concentration and heart rate measurement, therefore may aid in monitoring disease activity.

Objectives: The aim of this study is to evaluate physical activity in SSc patients by using a commercial smart band and investigate its association with clinical characteristics and patient-reported outcome measures of disease activity Methods: This prospective observational study included SSc patients with having a smartphone. Patients characteristics including age, sex, and organ involvements were recorded. Each participant was subjected to pulmonary function tests and 6MWT. All of patients answered Scleroderma Health Assessment Questionnaire (SHAQ, consisting of HAQ-Disability Index (DI) and visual analog scales (VAS) domains). All patients received Fitbit inspire HR smart band@ which records the number of steps, heart rate, distance and was instructed to wear it continuously for one week. Tracked data was collected from smartphones via Fitbit application

Results: Fifteen SSc patients (14 females and 1 male) participated in the study, $8(53.3 \%)$ had limited SSc and 7 (46.7) had diffuse SSc. The mean age was $48.5 \pm 15.5$ and the median disease duration was 4 (min-max:1-9) years. Eleven (73.3\%) patients had ILD and one patient had PAH. Musculoskeletal complaints were evident in two patients. Forced vital capacity (FVC, \% predicted), diffusion capacity of lung for carbon monoxide (DLCO, \%) in patients with ILD were significantly lower than patients without ILD median (IQR) 102 (30) vs 80 (27) p= $0.026,57$ (20) vs 95 (13), $p=0.002$, respectively. The median distance of 6MWTs were 450 (225) vs 568 (102) in ILD and non-ILD groups. The median total weekly step counts of ILD patients were remarkably lower in ILD patients compared to non-ILD 36.137 (17.879) vs 58.114 (80.681) steps/week, $(p=0.01)$. Patients with ILD had a bit higher median heart rate compared to non-ILD, 73 (9) vs 67.5 (12). The total weekly step counts were correlated with pulmonary function tests, including forced expiratory volume in one second (FEV1\%) $(r=0.57, p=0.025)$, FVC $(\%)(r=0.65, p=0.009)$, and DLCO $(\%)(r=0.70, p=0.005)$, patient-reported disease severity $(r=-0.66, p=0.007)$, and breathing problem $(r=-0.55, p=0.03)$ domains of SHAQ. There was no correlation between weekly step counts and 6MWT

Conclusion: The assessment of physical activity with smart activity bands may help to identify SSc patients with ILD. Tracked physical activity using smart bands correlates with pulmonary function tests and performs better than 6MWT, suggesting it as a useful tool for the assessment of disease activity.

References:

[1]Battaglia S, Bellia M, Serafino-Agrusa L, Giardina A, Messina M, Cannizzaro $\mathrm{F}$, et al. Physical capacity in performing daily activities is reduced in scleroderma patients with early lung involvement. Clin Respir J (2017) 11(1):36-42.

[2] Mainguy V, Provencher S, Maltais F, Malenfant S, Saey D. Assessment of daily life physical activities in pulmonary arterial hypertension. PLoS One (2011) 6(11):e27993.

Disclosure of Interests: None declared

DOI: 10.1136/annrheumdis-2020-eular.4641

\section{AB0549 \\ THE RELEVANCE OF AORTIC STENOSIS AND OUTCOME OF TAVI PROCEDURE FOR VALVE REPAIR IN SCLERODERMA PATIENTS}

A. Balbir-Gurman ${ }^{1}$, Y. Braun-Moscovici'. ${ }^{1}$ Rambam Health Care Campus, Rappaport Faculty of Medicine, Technion, Haifa, Israel

Background: Patients with systemic sclerosis (SSc) are at risk for developing aortic valve changes. The prevalence of aortic valve stenosis (AS) in SSc patients is unknown. Previously, patients with AS were treated with valve replacement; in recent years, Trans Catheter Aortic Valve Implantation (TAVI) is widely used in general population. The safety of Trans Catheter Aortic Valve Implantation (TAVI) in SSc has not been assessed.

Objectives: A retrospective study on aortic valve pathology and treatments including TAVI performance.
Methods: We reviewed 374 records of SSc patients at our site EUSTAR cohort and extracted cases with reported AS confirmed by ECHO cardiography and heart catheterization.

Results: We found data on 13 (3.4\%) patients with AS: 12 females (92.3\%) mean age 70.3 (SD 7.7) years, disease duration 15.4 (SD 6.3) years. Ten patients had limited SSc $(76.9 \%$, all cared anti-centromere antibodies) and 3 diffuse SSc (1 patient had RNAP3 and 2 had anti-topoisomerase antibodies); 5 (38.5\%) patients had significant coronary disease (3 underwent CABG, 2 had several PTCA). Eight (61.5\%) patients dead during years 2004 2019. Aortic valve replacement was performed in 5 patients $(4-$ metal and 1 - biological); 2 patients did not undergo AS repair due to impaired general condition; 6 patients underwent TAVI between January 2013 and December 2019 (5 at Rambam Cardiology Institute). All SSc patients underwent trans femoral TAVI under conscious sedation. The procedure was successful in all patients. The length of hospitalization was $5-14$ days (mean 8.2 days); 3 $(50 \%)$ patients needed pacemaker implantation (they did not have previous conduction abnormalities). The follow-up duration after TAVI was between 5 and 67 months (mean 20.7). During follow-up one patient developed bacterial endocarditis related to pacemaker device two months after the procedure the event resolved after removing the device and according antibiotics treatment; the same patient had transient ischemic attack two years later and another pacemaker implantation 3 years later due to complete AV block. One patients dead from urosepsis 11 months after TAVI, the death was not related to procedure. One patient developed anemia due to large hematoma after the procedure.

Conclusion: Screening for aortic valve pathology is essential as AS is not rare in SSc patients especially in those with long standing limited disease and positivity to centromere antibodies. AS in SSc patients may be associated with clinically significant coronary artery disease. TAVI was safe in our SSc patients without in-hospital mortality and benign long-term outcome.

Disclosure of Interests: Alexandra Balbir-Gurman Consultant of: Novartis, Yolanda Braun-Moscovici: None declared

DOI: 10.1136/annrheumdis-2020-eular.6372

\begin{tabular}{|l|l|}
\hline AB0550 & DIFFUSING CAPACITY OF THE LUNG FOR CARBON \\
MONOXIDE (DLCO) VS FORCED VITAL CAPACITY \\
(FVC): SYSTEMATIC LITERATURE REVIEW AND META- \\
ANALYSIS TO EXAMINE THEIR ABILITY TO MEASURE \\
CHANGE IN CLINICAL TRIALS IN SYSTEMIC \\
SCLEROSIS (SSC)
\end{tabular}

S. Bellando Randone ${ }^{1}$, E. Cappellini ${ }^{2}$, L. Nidiaci ${ }^{2}$, G. Lepri ${ }^{1}$, M. G. Lazzaroni ${ }^{3}$, C. Campochiaro ${ }^{4}$, G. Bagnato ${ }^{5}$, D. Sambataro ${ }^{6}$, G. Sambataro ${ }^{6}$, M. MatucciCerinic $^{1}$, D. Furst ${ }^{7} .{ }^{1}$ Department of Experimental and Clinical Medicine, University of Florence, Division of Rheumatology AOUC, Florence, Italy ${ }^{2}$ University of Florence, Florence, Italy; ${ }^{3}$ Rheumatology and Clinical Immunology, ASST Spedali Civili of Brescia, University of Brescia, Brescia, Italy; ${ }^{4}$ Unit of Immunology, Rheumatology, Allergy and Rare Diseases (UnIRAR), San Raffaele Scientific Institute, Vita-Salute San Raffaele University, Milan, Italy; ${ }^{5}$ P.O. G. Fogliani, ASP 5, Milazzo, Messina, Italy; ${ }^{6}$ University of Catania, Catania, Italy; ${ }^{7}$ Los Angeles, USA UCLA (emeritus); University of Washington, Seattle, Washington, Los Angeles, United States of America

Background: Lung involvement remains the main cause of morbidity and mortality in SSc. In 1 year clinical trials to assess lung involvement, FVC is usually evaluated and changes while the DLCO usually remains unchanged. In longer term observational studies, the DLCO often changes more than the FVC.

Objectives: To examine, through a systematic literature review(SLR) and meta-analysis, whether DLCO\%pred or FVC\%pred (both to be designated solely as DLCO and FVC henceforth), responds more in assessing SSC interstitial lung disease in first year and longer term follow-up(FU).

Table 1. Results from Random Effect Meta-Analysis

\begin{tabular}{|c|c|c|c|c|c|c|c|c|}
\hline & \multicolumn{3}{|c|}{$\begin{array}{c}21 \\
\text { studies }\end{array}$} & \multicolumn{4}{|c|}{$\begin{array}{c}5 \\
\text { studies }\end{array}$} & \\
\hline & $\begin{array}{l}\text { FVC } \% \\
\text { pred } \\
\text { mean } \\
\text { value }\end{array}$ & SE & $\begin{array}{c}\text { DLCO \% pred } \\
\text { mean value }\end{array}$ & SE & $\begin{array}{c}\text { FVC } \% \\
\text { pred } \\
\text { mean } \\
\text { value }\end{array}$ & SE & $\begin{array}{c}\text { DLCO \% pred } \\
\text { mean value }\end{array}$ & SE \\
\hline BASELINE & 78.8 & 3.43 & 59.7 & 5.52 & 81.1 & 5.68 & 55.6 & 11.59 \\
\hline 1 YEAR FOLLOW UP & 81.5 & 3.85 & 63.4 & 6.07 & - & - & - & - \\
\hline $\begin{array}{l}>1 \text { YEAR FOLLOW } \\
\text { UP }\end{array}$ & - & - & - & - & 83.4 & 4.68 & 54.7 & 12.37 \\
\hline
\end{tabular}

